\title{
AVALIAÇÃO DA QUALIDADE DE VIDA DE ALUNOS DE MEDICINA EM UMA UNIVERSIDADE PROMOTORA DA SAÚDE
}

\author{
Luana Carolina Rodrigues Guimarães, *Carolina Cristina de Freitas, Lilian Cristina Gomes do \\ Nascimento and Raquel Alves dos Santos
}

Programa de Pós Graduação em Promoção de Saúde da Universidade de Franca - Unifran

\section{ARTICLE INFO}

\section{Article History:}

Received $10^{\text {th }}$ April, 2021

Received in revised form

$28^{\text {th }}$ May, 2021

Accepted $08^{\text {th }}$ June, 2021

Published online $30^{\text {th }}$ July, 2021

\section{Key Words:}

Estudantes,

Promoção da Saúde, Saúde

*Corresponding author:

Carolina Cristina de Freitas

\begin{abstract}
A má qualidade de vida entre os estudantes de medicina está associada a um estilo de vida não saudável e pelo estresse emocional sofrido pelo excesso das atividades acadêmicas, o que futuramente pode levar diversas complicações de saúde desses discentes. O presente artigo é o resultado de uma pesquisa teve como objetivo avaliar a qualidade de vida dos alunos de Medicina em uma universidade promotora da saúde. Os dados foram obtidos no ano de 2018, por meio da aplicação um instrumento validado para verificar a qualidade de vida, nomeado de WHOQOLBREF. Participaram 166 universitários, com média de idade 21,29 anos. Os resultados mostraram qualidade de vida regular e necessidade de melhora na satisfação com a saúde, conforme orientado na classificação de Fleck. Correlacionando os diferentes domínios foi possível verificar que tanto o ambiente quanto a relação social impactam moderadamente no domínio psicológico. Conclui-se com esses resultados que a necessidade de sistemas de suporte para esses alunos, além da manutenção e ampliação de ambientes saudáveis nas universidades, a fim de poder relacionar todos os domínios com o ambiente, o que pode repercutir diretamente na qualidade de vida dos alunos.
\end{abstract}

Copyright (C) 2021, Luana Carolina Rodrigues Guimarães. This is an open access article distributed under the Creative Commons Attribution License, which permits unrestricted use, distribution, and reproduction in any medium, provided the original work is properly cited.

Citation: Luana Carolina Rodrigues Guimarães, Carolina Cristina de Freitas, Lilian Cristina Gomes do Nascimento and Raquel Alves dos Santos.

“Avaliação da qualidade de vida de alunos de medicina em uma universidade promotora da saúde”, International Journal of Development Research, 11, (07), 49112-49117.

\section{INTRODUCTION}

Qualidade de vida (QV) é um termo abrangente, multidimensional e subjetivo (Bampi et al., 2013) que envolve aspectos físicos, psicológicos e sociais que discorrem diretamente com a saúde (Almeida et al., 2012). Segundo a OMS a QV é "a percepção do indivíduo de sua posição na vida, no contexto da cultura e sistema de valores nos quais ele vive e em relação aos seus objetivos, expectativas, padrões e preocupações". Cleary e Wilson (1995) pontuam: "Refere-se aos vários aspectos da vida de uma pessoa que são afetados por mudanças no seu estado de saúde, e que são significativos para a sua qualidade de vida". Patrick e Erickson (1993 apud MINAYO; HARTZ; BUSS, 2000) "É o valor atribuído à duração da vida, modificado pelos prejuízos, estados funcionais e oportunidades sociais que são influenciados por doença, dano, tratamento ou políticas de saúde". A QV dos universitários vem sendo discutida recentemente, de forma lenta e progressiva (Catunda; Ruiz, 2008; Almeida; Gutierrez; Marques, 2012), uma vez que esse grupo normalmente está numa fase de mudança de vida, quando muitos saem de casa, e passam a ter novos afazeres e responsabilidades, podendo essas mudanças serem vistas de forma positiva ou negativa em suas vidas (JOIA, 2010). Andrade et al., (2016) consideram essa fase sendo como vulnerável, visto que os alunos são expostos a vários fatores de risco, como estresse, pressão psicológica, álcool, drogas entre outros. Os discentes que cursam Medicina têm uma particularidade em relação a essa carga de estresse e de pressão, já que mesmo antes de ingressar na universidade, o próprio vestibular de medicina é para eles uma fase difícil e concorrida (Aguiar, 2009; Pagnin; Queiroz, 2015). E normalmente segue em toda universidade e carreira médica; há dados que correlacionam que $51 \%$ dos médicos sofrem de algum distúrbio psicológico, segundo o conselho regional de medicina do Estado São Paulo (Barbosa et al., 2007; Serinolli; Novaretti, 2017). Apesar desse cenário complexo em relação à QV dos graduandos em Medicina, e ainda, em relação a outros cursos de graduação, existe um movimento recente que se iniciou no Brasil nos últimos 10 anos, e que tem como objetivo o alinhamento das universidades com práticas saudáveis, que são as Universidades Promotoras da Saúde (UPS), as quais integram políticas, estratégias e metas que vinculam o bem-estar do aluno, preocupando-se com a qualidade de vida dos discentes, com o ambiente no qual eles estão inseridos, e como estão vivendo dentro das universidades, colocando 
em voga a parte psicológica, social e até física do discente (Muñoz; Cabieses, 2008). Diante dessas considerações, o objetivo do presente estudo foi avaliar a QV de alunos do primeiro e segundo ano do curso de Medicina em uma UPS, usando questionário como instrumento de avaliação. Também foi estabelecida a correlação entre a percepção da qualidade de vida global e a satisfação com a saúde dos estudantes comparando e correlacionando os domínios físicos, psicológicos, relações sociais e meio ambiente a fim de verificar o quanto um domínio pode influenciar no outro em relação à qualidade de vida dos discentes.

\section{MATERIAIS E MÉTODOS}

Delineamento de pesquisa e aspectos éticos: A pesquisa foi aprovada pelo Comitê de Ética em Pesquisa (CEP) da Universidade de Franca/SP com parecer $n^{\circ} 2.930 .959$, de acordo com os aspectos éticos determinados pela Resolução 466/12, do Conselho Nacional de Saúde, resguardando os direitos éticos em pesquisas com seres humanos. Trata-se de um estudo observacional, transversal, descritivo e analítico da qualidade de vida dos discentes do primeiro e segundo anos do curso de Medicina de uma Universidade Promotora da Saúde, por meio da aplicação do questionário de qualidade de vida WHOQOL-BREF (FLECK, 2000). O convite para participação do estudo foi realizado de forma oral, em sala de aula, e foram explicados os objetivos do trabalho bem como as etapas de aplicação do questionário. Também foi apresentado o Termo de Consentimento Livre e Esclarecido (TCLE) para ser assinado pelos participantes da pesquisa.

Coleta de dados: Com base na quantidade de discentes matriculados no primeiro e segundo ano do curso de Medicina, considerando 95\% de confiança e erro padrão máximo igual a $5 \%$, foi obtida uma amostra aleatória e estratificada por período. Foram convidados a participar da presente pesquisa 218 alunos regularmente matriculados no curso de Medicina de uma Universidade que integra a Rede Iberoamericana das Universidades Promotoras da Saúde, sendo 106 discentes do primeiro ano de medicina e 112 do segundo ano. Foram critérios de não inclusão os universitários que usam regularmente medicamentos como ansiolíticos, antidepressivos, psicotrópicos; uso recente (três dias) de drogas ilícitas, os menores de 18 anos, maiores de 60 anos e pessoas com deficiência física; e os critérios de exclusão foram os que preencheram de forma incorreta os questionários durante a pesquisa. Assim, não foram incluídos 23 (10,5\%) participantes do primeiro ano, desses 18 do sexo feminino e cinco masculino, e $20(9,1 \%)$ participantes do segundo ano, sendo 14 do sexo feminino e seis masculino, e foram excluídos nove participantes no total. Para aplicação do questionário os estudantes foram dispostos em uma sala de aula, sendo no máximo dez alunos em cada sala. Após uma explicação prévia, os estudantes responderam individualmente o questionário WHOOL-BREF, constituído de questões que abrangem os domínios físico (sete questões), psicológico (seis questões), relações sociais (três questões) e meio ambiente (oito questões). Os resultados são apresentados em scores (FLECK, 2000).

Tratamento dos dados e análise estatística: A análise estatística deste estudo foi descritiva e inferencial. As variáveis independentes foram aquelas relacionadas ao perfil sociodemográfico (sexo e idade) e ao ano do curso de medicina. A variável categórica sexo foi descrita por sua frequência absoluta e relativa de acordo com o ano de curso do estudante. Em seguida a estatística inferencial foi efetuada por meio do teste do Qui-quadrado $\left(\mathrm{x}^{2}\right)$ para comparação de frequências. Após isso aplicou-se testes de normalidade e de homocedasticidade afim de prosseguir ou não com a análise paramétrica das médias da idade. Assim, a variável quantitativa idade foi descrita por meio de suas medidas de tendência central média e de dispersão: desviopadrão, valores máximos e mínimos. Então foi efetuada a estatística inferencial com o teste T não pareado para comparação de médias. As variáveis dependentes do estudo foram compostas pelos 4 domínios e pelas 26 perguntas (facetas) do questionário WHOQOL-BREF. O nível de satisfação para cada faceta do questionário foi baseado na escala Likert de 5 pontos: 1: muito ruim, 2: ruim, 3: nem bom e nem ruim (neutro), 4: bom e 5: muito bom. As pontuações de 1 a 5 na escala Likert para cada faceta e para cada domínio foram classificadas de acordo com a publicação de Marcelo Fleck, cujo resultados que variam de 1 até 2,9 necessitam melhorar, de 3 até 3,9 estão regulares, de 4 até 4,9 estão boas e 5 estão muito boas (FLECK et al., 2000). Estas pontuações para cada faceta e para cada domínio WHOQOLBREF foram descritas por meio de suas frequências relativas (em porcentagem a partir da frequência de participantes que responderam de 1 a 5) e das medidas de tendência central médias e medianas assim como de suas medidas de dispersão: desvio-padrão, coeficiente de variação, valores máximos, mínimos e amplitude. Então verificou-se a distribuição normal e a homocedasticidade destas variáveis numéricas, sendo então submetidas ao teste não paramétrico de Kruskal Wallis. As médias das pontuações das facetas gerais de percepção de qualidade de vida (pergunta 1) e da satisfação com a saúde (pergunta 2) foram correlacionadas entre si por meio do Teste de correlação R de Pearson a fim de averiguar o quanto uma faceta pode influenciar na outra faceta. Em seguida elaborou-se uma matriz de correlação entre os quatro domínios WHOQOL-BREF também por meio do teste de correlação $R$ de Pearson. Os valores de correlação foram classificados de acordo com os critérios estabelecidos por Mitra (1999) valores entre 0,20 e 0,39 - fraca correlação; entre 0,40 e 0,59 - moderada correlação; acima de 0,60 - forte correlação. Os dados foram organizados em planilhas e os gráficos foram elaborados com a ajuda do programa Excel da Microsoft. Todas as análises estatísticas consideraram o nível de significância de 5\% (com grau de significação de $\mathrm{p}<0,05)$.

\section{RESULTADOS}

Perfil sociodemográfico dos participantes: A amostra foi constituída de 166 participantes, sendo 83 (50\%) do primeiro ano e 83 $(50 \%)$ do segundo ano. Matriculados no primeiro ano do curso, $67,4 \%$ eram do sexo feminino $(\mathrm{n}=56)$ e $35,5 \%$ do sexo masculino $(n=27)$. $O$ teste $X^{2}$ para comparação de frequências não demonstrou diferenças estatísticas entre a distribuição dos sexos entre os anos $\left(\mathrm{X}^{2}=0,424 ; \mathrm{p}=0,515\right)$. A média de idade dos discentes do primeiro ano foi de $20,9 \pm 3,1$ e $21,19 \pm 2,56$ anos para os indivíduos do sexo feminino e masculino, respectivamente $(\mathrm{t}=0,266 ; \mathrm{p}=0,7913)$. Em relação ao segundo ano, $62,6 \%$ eram do sexo feminino $(n=52)$ e $37,3 \%(\mathrm{n}=31)$ do sexo masculino com idade média de $20,9 \pm 1,4$ e $22,2 \pm 3,6$ anos, respectivamente $(\mathrm{t}=2,236 ; \mathrm{p}=0,03)$. Portanto os indivíduos do sexo masculino apresentavam idade superior a das mulheres do segundo ano, conforme Tabela 1.

Correlação entre a percepção da qualidade de vida e a satisfação com a saúde: No questionário sobre a qualidade de vida, com as facetas 1 e 2 foi possível avaliar a percepção da qualidade de vida (pergunta 1) e a satisfação com a saúde (pergunta 2). A média dos resultados encontrados foi de 3,5 na faceta 1 e 2,9 na faceta 2 , como pode ser visualizado na Tabela 2 . O que significa que a qualidade de vida está regular entre os estudantes e a satisfação com a saúde necessita melhorar, segundo a classificação feita por Fleck (2000). A Tabela 2 também apresenta resultados que demonstram que houve uma correlação moderada e positiva de $0,474(p<0,001)$ entre a percepção de qualidade de vida e a satisfação com a saúde. Ou seja, segundo os participantes, a qualidade de vida pode impactar diretamente a satisfação com a própria saúde. Portanto, ao melhorarem a qualidade de vida, a saúde dos participantes também pode ser melhorada. Também foi verificado que a variabilidade foi baixa entre as respostas, pois o coeficiente de variação $(\mathrm{CV})$ foi menor do que $50 \%$, o que demonstra que os dados foram homogêneos.

Descrição, comparação e correlação entre os domínios do questionário WHOQOL-BREF: Devido ao fato das respostas para cada faceta dos domínios do questionário do WHOQOL-BREF serem por meio da escala Likert, não seria apropriada a descrição apenas por média devido a sua distribuição não normal. 
Assim optou-se por demonstrar os resultados por meio do gráfico radar de medianas. Em relação ao domínio físico, as facetas que apresentaram as maiores medianas foram sono e repouso bem como a mobilidade, ambas com mediana igual a 4 (bom). Em relação a esse domínio, $62,7 \%$ dos participantes relataram ter sono e repouso bons ou muito bons. Além do mais, $79,5 \%$ dos participantes tinham boa ou muito boa mobilidade. Ao passo que as facetas relacionadas à energia e fadiga, bem como dor e desconforto, tiveram a menor mediana de 2 (ruim). Nessas facetas $71,6 \%$ dos estudantes responderam ruim ou muito ruim para a energia e fadiga. Este percentual foi semelhante aos $71 \%$ dos participantes que também responderam ruim ou muito ruim para dor e desconforto. Embora o sono, repouso e mobilidade estivessem bons, a energia, fadiga, dores e desconfortos estavam regulares, segundo a classificação de Fleck (2000). As facetas capacidade de trabalho, dependência de medicação ou tratamentos e atividades da vida cotidiana também estiveram regulares, com mediana de 3 (nem boa e nem ruins), conforme demonstrado na Figura 1. As facetas do domínio psicológico: sentimentos positivos, sentimentos negativos, auto estima e o pensar, aprender, memória e concentração apresentaram mediana de 3 (nem bons e nem ruins) e representaram as menores medianas deste domínio. De acordo com os resultados $62,5 \%$ dos participantes estavam neutros em relação aos sentimentos positivos. Esta frequência foi maior do que os $48,2 \%$ dos estudantes que estavam neutros e $24,1 \%$ que relatavam ter sentimentos negativos.

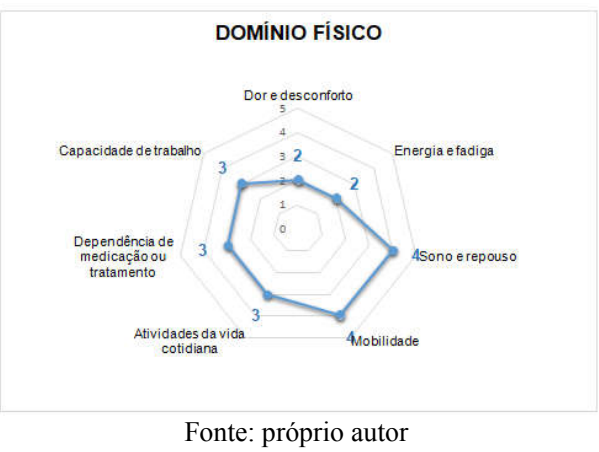

Figura 1. Mediana das facetas do domínio físico do WHOQOLBREF

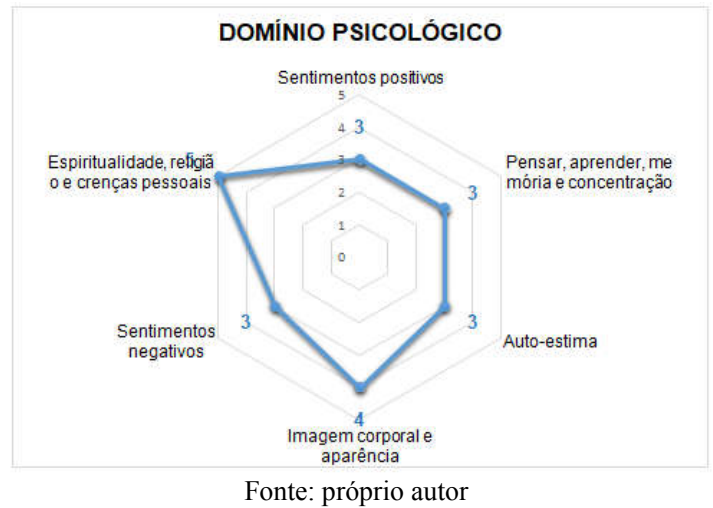

Figura 2. Mediana das facetas do domínio psicológico do WHOQOL-BREF

Portanto, de acordo com os resultados obtidos no grupo de estudo, os sentimentos positivos foram mais frequentes do que os negativos. Porém isso não afetou o pensar, o aprender, a memória e a concentração, já que $47,1 \%$ dos participantes consideravam que esta faceta estivesse boa ou muito boa em sua vida. A autoestima também apresentou a mediana de 3 (nem boa e nem ruim), pois 46,4\% dos participantes assinalaram esta resposta na escala Likert. Embora a satisfação com a imagem corporal e aparência estivessem boas (mediana de 4), a faceta que recebeu a melhor classificação foi a espiritualidade, religião e crenças pessoais com mediana de 5 (muito boa), pois $81,9 \%$ dos participantes acreditavam que esta faceta em sua vida estaria boa ou muito boa, conforme a Figura 2.

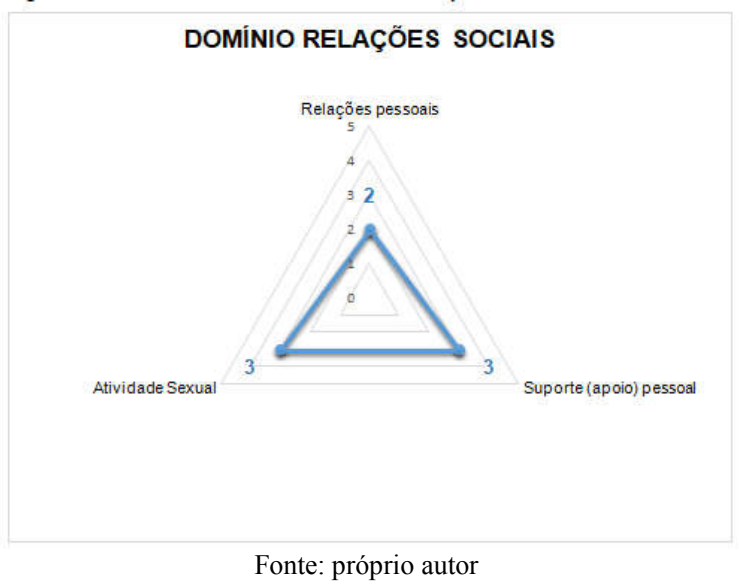

Figura 3. Mediana das facetas do domínio relações sociais do WHOQOL-BREF

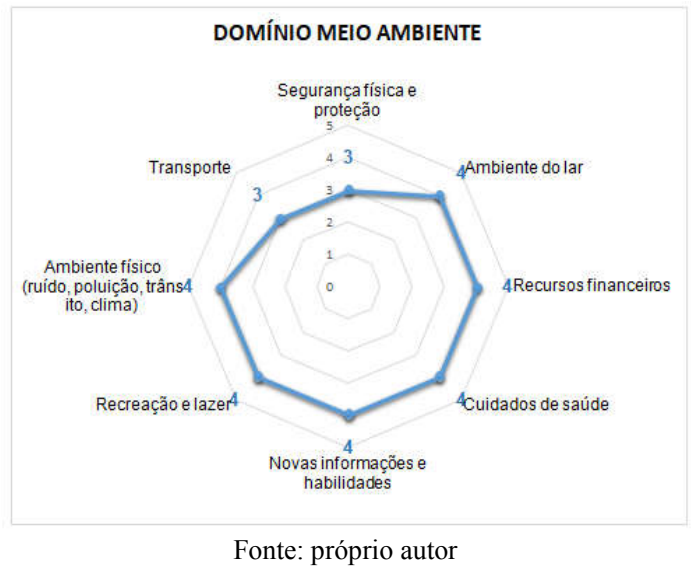

Figura 4. Mediana das facetas do domínio meio ambiente do WHOQOL-BREF

As relações pessoais fazem parte do domínio relações sociais e apresentou mediana de 2 , ou seja, classificação de ruim na escala Likert do WHOQOL-BREF. Visto que 53,6\% dos estudantes relataram que esta faceta necessitava melhorar em suas vidas. O suporte (apoio) pessoal apresentou mediana de 3, pois 47,6\% dos participantes assinalaram que o apoio pessoal nesta fase de vida não estava bom e nem ruim. A atividade sexual também apresentou mediana de 3 , já que $54,2 \%$ dos entrevistados responderam que esta faceta não estava nem boa e nem ruim, como demonstrado na Figura 3. As facetas do domínio meio ambiente apresentaram as maiores medianas em relação às facetas dos demais domínios. Embora a faceta segurança física e proteção bem como a faceta transporte apresentaram a mediana de 3 (nem bom e nem ruim), as demais facetas apresentaram mediana de 4 (bom). A frequência relativa dos discentes que responderam ruim e neutro para a segurança física e o transporte foi, respectivamente de $57 \%$ e $49,4 \%$.

Já a frequência relativa das respostas de bom a muito bom das facetas ambiente do lar, recursos financeiros, cuidados de saúde e sociais, oportunidades de adquirir novas informações, oportunidade de recreação e lazer assim como ambiente físico foram de $66,3 \%, 57,3 \%$, $81,3 \%, 75,9 \%, 65,7 \%$, e $66,2 \%$ respectivamente. Assim os estudantes de medicina responderam que, das facetas do domínio meio ambiente, a que eles estavam mais satisfeitos era a dos cuidados de saúde e sociais, como pode ser observado nas medianas apresentadas na Figura 4. A classificação de Fleck (2000) para os domínios físicos (média de 2,98) e para as relações sociais (média de 2,79) demonstrou que são domínios que necessitavam melhorar. Já os domínios psicológicos (média de 3,44) e de meio ambiente (média de 3,67) estavam regulares. Então, comparou-se as medianas dos domínios do questionário WHOQOL-BREF obtidos a partir das medianas de cada uma de suas facetas por meio do teste não-paramétrico de Kruskal Wallis. 
Tabela 1. Análise descritiva das variáveis sociodemográficas e comparação entre as médias de idades de acordo com o sexo e o ano regularmente matriculado dos participantes

\begin{tabular}{|c|c|c|c|c|c|c|c|}
\hline Variáveis sociodemográficas & Média & Mediana & $\begin{array}{l}\text { Desvio } \\
\text { Padrão }\end{array}$ & $\begin{array}{l}\text { Valor } \\
\text { Mínimo }\end{array}$ & $\begin{array}{l}\text { Valor } \\
\text { Máximo }\end{array}$ & Teste T & Valor $\mathrm{p}$ \\
\hline \multicolumn{8}{|l|}{ Primeiro ano } \\
\hline Feminino & 20,9 & 20,0 & 3,1 & 18 & 38 & 0,266 & 0,7913 \\
\hline Masculino & 21,1 & 21,0 & 2,5 & 18 & 28 & & \\
\hline \multicolumn{8}{|l|}{ Segundo ano } \\
\hline Feminino & 20,9 & 21,0 & 1,4 & 18 & 25 & 2,236 & $0,03 *$ \\
\hline Masculino & 22,2 & 21,0 & 3,6 & 19 & 38 & & \\
\hline
\end{tabular}

*Apresentou diferenças estatísticas $(\mathrm{p}<0,05)$. Fonte: próprio autor

Tabela 2. Descrição e correlação da percepção da qualidade de vida com a satisfação com a saúde

\begin{tabular}{|c|c|c|c|c|c|c|c|c|}
\hline \multirow[t]{2}{*}{ Questões } & \multirow[t]{2}{*}{ Mediana } & \multirow[t]{2}{*}{ Média } & \multirow[t]{2}{*}{ DP } & \multirow[t]{2}{*}{$\mathrm{CV}$} & \multirow{2}{*}{$\begin{array}{l}\text { Valor } \\
\text { mínimo }\end{array}$} & \multirow{2}{*}{$\begin{array}{l}\text { Valor } \\
\text { máximo }\end{array}$} & \multirow[b]{2}{*}{$\begin{array}{l}\text { Correlaçãode } \\
\text { Pearson }\end{array}$} & \multirow[b]{2}{*}{ Valor $\mathrm{p}$} \\
\hline & & & & & & & & \\
\hline Qualidade de Vida (Q1) & 4 & 3,50 & 0,85 & 24,31 & 1 & 5 & & \\
\hline $\begin{array}{l}\text { Satisfação com a saúde } \\
\text { (Q2) }\end{array}$ & 3 & 2,90 & 0,92 & 31,55 & 1 & 5 & 0,474 & $<0,001^{*}$ \\
\hline
\end{tabular}

Legenda: Q1, questão um; Q2, questão dois; DP, desvio-padrão; CV, coeficiente de variação. *Apresentou diferenças estatísticas (p<0,05).

Fonte: próprio autor

Tabela 3. Comparação entre as medianas dos domínios do WHOQOL-BREF

\begin{tabular}{lllllll}
\hline Domínios & Média & $\begin{array}{l}\text { Classificação por } \\
\text { Marcelo } \\
(2000)\end{array}$ & Mediana & Valor Mínimo & Valor Máximo & Kruskal Wallis \\
\hline Físicos & 2,9 & Necessita melhorar & 3 & 2 & 4 & 6,624 \\
Psicológicos & 3,4 & Regular & 3 & 3 & 3 & 0,08 \\
Relações Sociais & 2,7 & Necessita Melhorar & 3 & 2 & 3 & \\
Meio Ambiente & 3,6 & Regular & 4 & 3 & 4 & \\
\hline
\end{tabular}

Fonte: próprio autor.

Tabela 4. Matriz de correlação entre os domínios do questionário WHOQOL-BREF

\begin{tabular}{|c|c|c|c|c|}
\hline Domínios & Físicos & Psicológicos & Relações sociais & Meio Ambiente \\
\hline Físicos & 1 & $\begin{array}{l}0,390 \\
p<0,001 *\end{array}$ & $\begin{array}{l}0,365 \\
\mathrm{p}<0,001 *\end{array}$ & $\begin{array}{l}0,380 \\
\mathrm{p}<0,001 *\end{array}$ \\
\hline Psicológicos & & 1 & $\begin{array}{l}\mathbf{0 , 5 1 6} \\
\mathrm{p}<0,001 *\end{array}$ & $\begin{array}{l}\mathbf{0 , 5 9 0} \\
\mathrm{p}<0,001 *\end{array}$ \\
\hline Relações Sociais & & & 1 & $\begin{array}{l}0,398 \\
\mathrm{p}<0,001 *\end{array}$ \\
\hline Meio ambiente & & & & 1 \\
\hline
\end{tabular}

Assim foi verificado que se deve aceitar a hipótese nula de que não há diferenças entre as medianas dos diferentes domínios $(\mathrm{p}=0,08)$, conforme Tabela 3 . A fim de se correlacionarem os quatro domínios entre si, com o objetivo de verificar o quanto a satisfação com um domínio da qualidade de vida pode impactar em outro domínio, aplicou-se o teste de correlação de Pearson para as médias obtidas a partir das diferentes facetas que compõem cada domínio. Verificou-se que todos os domínios se correlacionam entre si $(\mathrm{p}<0,001)$.

O domínio físico apresentou correlação fraca de 0,390, 0,365 e de 0,380 com os domínios psicológicos, relações sociais e meio ambiente, respectivamente $(\mathrm{p}<0,001)$. Já o domínio psicológico apresentou correlação moderada de 0,516 e 0,590 com os domínios relações sociais e meio ambiente. O que demonstrou que as relações sociais e o meio ambiente impactavam significativamente o domínio psicológico do estudante $(\mathrm{p}<0,001)$. O domínio relações sociais também apresentou correlação fraca e significativa de 0,398 com o meio ambiente $(p<0,05)$, Tabela 4 .

\section{DISCUSSÃo}

Este estudo demonstrou que a maior parte dos participantes do primeiro e segundo ano do curso de Medicina da UPS a qual pertence era do sexo feminino com faixa etária média de 21, $22 \pm 2,77$ anos. Percebe-se um maior número de participantes do sexo feminino, e os dados do Censo (BRASIL, 2010) corroboram com este achado, pois descrevem o fato de se ter a nível nacional um maior número de mulheres cursando o ensino superior. Costa e colaboradores (2020) obteve resultados semelhantes, com $50,5 \%$ dos participantes do sexo feminino, e a idade média deles de 22,9. Assim também, um estudo realizado no estado do Piauí também mostrou predominância de mulheres de 61,5\% (Oliveira, 2018). Uma metanálise realizada no Brasil observou que estudantes do sexo feminino apresentaram notas mais baixas no questionário quando comparados ao do sexo masculino (SOLIS; LOTUFO-NETO, 2019). Domantay (2014) observou o sexo feminino mais vulnerável e com menor qualidade de vida. Solis e Lotufo-Neto (2019), também destacaram domínios físicos e psicológicos mais afetados em relação aos demais domínios. 
No presente estudo, o domínio físico teve média inferior ao psicológico, segundo a classificação de Fleck (2000), demonstrando assim a necessidade de melhora. Chazan, Campos e Portugal (2015) também observaram os domínios físico e psicológico mais afetados nos alunos de medicina da Universidade Federal do Rio de Janeiro. Estudo feito com a mesma população, porém com uma amostra maior, de alunos de primeiro ao quarto ano, evidenciou uma melhora no domínio psicológico com o avançar da graduação, sendo também detectada melhora nos demais domínios conforme os anos avançam, fato esse explicado pelo choque do aluno ao ingressar na universidade e adaptação do mesmo com o passar do tempo (Andrade et al., 2019). As duas primeiras facetas do questionário WHOQOL-BREF: percepção de qualidade de vida e satisfação com a saúde, pela classificação de Fleck (2000) estava regular e necessita melhorar, respectivamente. Além do mais, estavam correlacionadas entre si, ou seja, os estudantes acreditavam que a melhora na qualidade de vida também poderia trazer uma melhorara na situação de saúde $(\mathrm{p}<0,05)$. Um estudo com os alunos da Universidade de Brasília evidenciou que $71,5 \%$ dos participantes avaliaram sua qualidade de vida como boa ou muito boa, e $70,2 \%$ estavam satisfeitos ou muito satisfeitos com a saúde (BAMPI et al., 2013). Alves et al., (2010) também mostraram a QV e a satisfação com a saúde boa ou muito boa, em um estudo realizado com 100 acadêmicos de Medicina em Sorocaba no Estado de São Paulo. Em relação ao domínio físico, as facetas dor e desconforto, além da energia e fadiga, apresentaram medianas baixas. As atividades da vida cotidiana, dependência de medicação ou de tratamento médico e capacidade de trabalho estavam regulares, embora o sono, repouso e a mobilidade pudessem ser boas. NogueiraMartins (2010), em um estudo realizado com médicos residentes, evidenciou que o sono, cansaço, estresse, fadiga estava presente na maioria dos participantes. Médicos residentes têm uma má qualidade de vida, sonolência diurna e Burnout com maior frequência quando comparados a outros grupos de pessoas (Asaiag et al., 2010). Barros e colaboradores também evidenciaram a parte física comprometida, com privação do sono, longas jornadas e estresse em universitários da área da saúde. De acordo com Aziz et al., (2020) existe uma forte relação entre o domínio físico e psicológico; o domínio físico é um fator positivo no controle de depressão, oscilação de humor, nervosismo dentre outros. O comportamento sedentário, devido aos longos períodos de estudo demandados na educação em medicina, está associado a uma má qualidade de vida física. Já em relação ao domínio psicológico, a espiritualidade, religião e crenças pessoais eram consideradas muito boas. A imagem corporal e aparência eram boas, porém a autoestima, os sentimentos negativos, o pensar, aprender, a memória e concentração bem como os sentimentos positivos apresentaram-se regulares. Bampi (2013) teve como resultado, em um estudo realizado com alunos da Faculdade de Medicina da Universidade de Brasília, os sentimentos positivos, auto estima, aparência e crenças pessoais, com mediana 4, sendo boa; destes, $80 \%$ dos participantes responderam que a vida tinha sentido, porém, 95\% dos entrevistados apresentaram sentimentos negativos (mal humor, depressão, ansiedade, desespero).

Também foi verificado no presente estudo, que a satisfação relacionada com as relações pessoais, as quais apresentaram valores baixos, o suporte (apoio) pessoal e a atividade sexual dos participantes se apresentaram regulares, estas representam as facetas do domínio das relações sociais. Uma pesquisa realizada por Vo, Nguyen, Ta (2020) relata que estudantes que participaram de um estudo semelhante obtiveram maiores pontuações no domínio social em relação aos outros domínios, podendo indicar uma associação entre o desenvolvimento de relações sociais e de uma melhor QV para estudantes de medicina. Um estudo realizado em Portugal com alunos da área da saúde apresentou o domínio relação social baixo, principalmente com alunos do primeiro ano do curso, com reduzido tempo para o lazer e dificuldade de relação com família e amigos (Oliveira et al., 2016). O domínio meio ambiente obteve as maiores pontuações, as suas facetas, ambiente do lar, ambiente físico geral, recreação e lazer, novas informações e habilidades, cuidados de saúde e recursos financeiros foram classificados como bons, embora as facetas transporte e segurança física demonstraram regulares. Conforme Malibary et al., (2019), em um estudo realizado na Arábia
Saudita, também verificaram-se as maiores pontuações nas facetas ambientais. Pode ser observado que estes resultados dependem do currículo das universidades e uma menor pontuação da QV foi observada em diferentes anos da universidade, dependendo do programa. Pode também relacionar ao ambiente da Universidade Saudável como ponto positivo neste domínio, com esse estudo. Entretanto, ao comparar as medianas dos domínios, verificou-se que não houve diferenças estatísticas $(\mathrm{p}=0,08)$, pois, de acordo com a classificação de Fleck, todos os domínios necessitavam melhorar ou estavam regulares. Em relação aos diferentes domínios houve correlação entre eles $(p<0,001)$, sendo as maiores correlações, aquelas classificadas como moderadas entre os domínios psicológicos com as relações sociais e o meio ambiente.

\section{Conclusão}

Neste estudo pode ser observado que todos os quatro domínios da qualidade de vida do questionário WHOQOL-BREF necessitavam melhorar ou estavam regulares, embora esta classificação tenha variado entre as 26 facetas. A maior pontuação foi destacada para a espiritualidade, religião e crenças pessoais, enquanto a menor pontuação se destacou para a faceta de relações pessoais. Também pode ser observado que as relações sociais e o meio ambiente impactaram de forma significativamente moderada o domínio psicológico dos estudantes que participaram deste estudo, possivelmente por considerar o fato de que estes alunos estão constantemente sob estresse. Em consideração aos resultados obtidos por meio dos questionários aplicados, torna-se importante uma maior investigação destes dados, e a necessidade de sistemas de suporte eficientes para esses alunos, planejamento de estratégias de prevenção de desordens físicas e mentais, visando melhorar, principalmente, as relações sociais a fim de impactar o domínio psicológico e desta forma melhorar a qualidade de vida e a satisfação com a própria saúde dos discentes do curso de medicina da referida universidade. Destaca-se ainda a necessidade de manter e estender os ambientes saudáveis nas universidades a fim de poder relacionar todos os domínios com o ambiente, o que pode ter repercussão direta na qualidade de vida dos alunos.

\section{REFERÊNCIAS}

Aguiar, Sâmia Mustafa et al. Prevalência de sintomas de estresse nos estudantes de medicina. Jornal Brasileiro de Psiquiatria, v. 58, n. 1, p. 34-38, 2009.

Almeida, Marco Antonio Bettine de; GUTIERREZ, Gustavo Luis; MARQUES, Renato Francisco Rodrigues. Qualidade de vida: definição, conceitos e interfaces com outras áreas de pesquisa. 2012. São Paulo: Escola de Artes, Ciências e Humanidades EACH/USP, 2012.

Alves, João Guilherme Bezerra et al. Qualidade de vida em estudantes de Medicina no início e final do curso: avaliação pelo Whoqol-bref. Revista Brasileira de Educação Médica, v. 34, n. 1, p. 91-96, 2010.

Andrade, Ana Isabel Nunes Pereira de Azevedo; MARTINS, Rosa Maria Lopes. Funcionalidade familiar e qualidade de vida dos idosos. Millenium-Journal of Education, Technologies, and Health, n. 40, p. 185 $\square 199-185 \square 199,2016$.

Andrade, Felipe Kaluf et al. Qualidade de vida e burnout entre estudantes de medicina que vivenciam o método de Aprendizagem Baseada em Problemas. Aletheia, v. 52, n. 1, 2019.

Asaiag, Paulo Eduardo et al. Avaliação da qualidade de vida, sonolência diurna e burnout em médicos residentes. Revista Brasileira de Educação Médica, v. 34, n. 3, p. 422-429, 2010.

Aziz, Yasir et al. Quality of life of students of a private medical college. Pakistan Journal of Medical Sciences, v. 36, n. 2, p. 255, 2020.

Bampi, Luciana Neves da Silva et al. Qualidade de vida de estudantes de medicina da Universidade de Brasília. Revista Brasileira de Educação Médica, v. 37, n. 2, p. 217-225, 2013.

Barbosa, Genário Alves et al. A saúde dos médicos no Brasil. Brasília: Conselho Federal de Medicina, v. 220, 2007. 
Brasil. Censo demográfico 2010. Características gerais da população, religião e pessoas com deficiência. Rio de Janeiro: IBGE, 2012. Acompanha 1 CD-ROM.

Catunda, Maria Antunes Pinto; RUIZ, Valdete Maria. Qualidade de vida de universitários. Pensamento plural, v. 2, n. 1, p. 22-31, 2008.

Chazan, Ana Cláudia Santos; CAMPOS, Mônica Rodrigues; PORTUGAL, Flávia Batista. Qualidade de vida de estudantes de medicina da UERJ por meio do Whoqol-bref: uma abordagem multivariada. Ciência \& Saúde Coletiva, v. 20, p. 547-556, 2015.

Costa, Deyvison Soares da et al. Sintomas de Depressão, Ansiedade e Estresse em Estudantes de Medicina e Estratégias Institucionais de Enfrentamento. Revista Brasileira de Educação Médica, v. 44, n. 1,2020

Domantay, John Anthony A. Health-related quality of life of future physicians at a medical school in the Philippines: a crosssectional study. Sage Open, v. 4, n. 3, p. 2158244014545459, 2014.

Fleck, Marcelo et al. Aplicação da versão em português do instrumento abreviado de avaliação da qualidade de vida" WHOQOL-bref". Revista de saúde pública, v. 34, n. 2, p. 178$183,2000$.

Joia, Luciane Cristina. Perfil do estilo de vida individual entre estudantes universitários. Revista movimenta, v. 3, n. 1, p. 16-23, 2010.

Malibary, Husam et al. Quality of Life (QoL) among medical students in Saudi Arabia: a study using the WHOQOL-BREF instrument. BMC medical education, v. 19, n. 1, p. 344, 2019.

Minayo, Maria Cecília de Souza; HARTZ, Zulmira Maria de Araújo; BUSS, Paulo Marchiori. Qualidade de vida e saúde: um debate necessário. Ciência \& saúde coletiva, v. 5, p. 7-18, 2000.

Mitra, Ananda. Research methods in park, recreation, and leisure services. 1999.
Muñoz, Mónica; Cabieses, Baltica. Universidades y promoción de la salud:¿ cómo alcanzar el punto de encuentro?. Revista panamericana de salud pública, v. 24, p. 139-146, 2008.

Nogueira-Martins, Luiz Antonio. Qualidade de vida dos médicos residentes: revisão de estudos brasileiros. Cad abem, v. 6, p. 12-8, 2010.

Oliveira, Alexandra et al. Perceived quality of life among first-year health students engaged in problem based learning and traditional teaching model: First-year students of allied health sciences. In: 2016 11th Iberian Conference on Information Systems and Technologies (CISTI). IEEE, 2016. p. 1-7.

Oliveira, Ellaine Santana de. Estresse E Comportamentos De Risco À Saúde Entre Estudantes Universitários. 2018.

Pagnin, Daniel; QUEIROZ, Valeria. Comparison of quality of life between medical students and Young general populations. Education for Health, v. 28, n. 3, p. 209, 2015.

Serinolli, Mario Ivo; NOVARETTI, Marcia Cristina Zago. A crosssectional study of sociodemographic factors and their influence on quality of life in medical students at Sao Paulo, Brazil. PloS one, v. 12, n. 7, 2017.

Solis, Ana Cristina de Oliveira.; LOTUFO-NETO, Francisco. Predictors of quality of life in Brazilian medical students: a systematic review and meta-analysis. Brazilian Journal of Psychiatry, v. 41, n. 6, p. 556-567, 2019.

Vo, Trung Quang; NGUYEN, Hieu Thanh Thi; TA, Anh Phuong Ngoc. Effect of sociodemographic factors on quality of life of medical students in southern Vietnam: A survey using the WHOQOL-BREF assessment. Journal of Pharmacy \& Pharmacognosy Research, v. 8, n. 3, p. 211-224, 2020.

Wilson, Ira B.; Cleary, Paul D. Linking clinical variables with healthrelated quality of life: a conceptual model of patient outcomes. Jama, v. 273, n. 1, p. 59-65, 1995. 